\title{
STABLE VECTOR BUNDLES ON CURVES: NUMERICAL INVARIANTS OF THEIR EXTREMAL SUBBUNDLES
}

\author{
E. BALLICO
}

\begin{abstract}
Let $X$ be a smooth projective curve of genus $g \geq 2$ and $E$ a rank $r$ vector bundle on $X$. If $1 \leq t<r$ set $s_{t}(E):=\sup \{t(\operatorname{deg}(E))-r(\operatorname{deg}(F))$, where $F$ is a rank $t$ subsheaf of $E\}$. Here we construct rank $r$ stable vector bundles $E$ on $X$ such that the sequence $\left\{s_{t}(E)\right\}_{1 \leq t<r}$ has a prescribed value and the set of all subsheaves of $E$ with maximal degree may be explicitely described.
\end{abstract}

\section{Introduction}

Let $X$ be a smooth projective curve of genus $g \geq 2$ and $E$ a rank $r$ vector bundle on $X$. For every integer $t$ with $1 \leq t<r$, set $s_{t}(E):=\sup \{t(\operatorname{deg}(E))-$ $r(\operatorname{deg}(F))$, where $F$ is a rank $t$ subsheaf of $E$ \}. These integers are usually called the Lange invariants of $E$, since their use in [6]. They are important invariants of a stable vector bundle and give a stratification of the moduli scheme $M(X ; r, d)$ of all rank $r$ stable vector bundles on $X$ with degree $d$ (see [6] and [4]). The affermative solution of the so-called Lange conjecture proved in [10] or [3] or, under certain assumptions on $r$ and $g$, in [4], shows that all these strata, for fixed $t$, are non-empty (at least in characteristic 0). However, there should be further constraints on the orderered set of $r-1$ integers $\left(s_{1}(E), \ldots, s_{r-1}(E)\right)$ for $E \in M(X ; r, d)$. The aim of this paper is the construction of stable bundles with suitable Lange invariants. For a rank $r$ vector bundle $E$ we will consider related invariants $d_{t}(E), 1 \leq i \leq r-1$, with $d_{1}(E)=s_{1}(E)$ and $\sum_{1 \leq i \leq t} d_{i}(E) \leq s_{t}(E)$ for every $t$ (see Definition 1.1). The same construction will give the existence of stable bundles with suitable $\left(s_{1}(E), \ldots, s_{r-1}(E)\right)$. For a "general" member, $E$, of each strata of $M(X ; r, d)$ (or of each "good component of each strata") there is a unique rank $t$ subbundle $F$ of $E$ with $s_{t}(E)=t(\operatorname{deg}(E))-r(\operatorname{deg}(F))$ (see [4], [11] and [10]). Sometimes, our examples will have a unique such $F$; sometimes such $F$ will not be unique and we will describe explicitly all of them (see Remark 2.4). Our construction is characteristic free. It uses as an essential 
tool elementary transformations of vector bundles. A key ingredient is an idea contained in [10] (see Lemma 1.2). In this way it is easy to construct stable vector bundles with suitable filtrations or with high degree saturated subbundles which are direct sums of preassigned line bundles and hence with prescribed numerical properties (see the statements of Theorems 2.2, 2.5, 2.6, 2.7, 2.8, 2.9 and 2.10). We obtain in this way also stable extensions, $F$, of two very particular vector bundles $G$ and $H$ (even unstable ones) (see Theorem 1.9).

The author was partially supported by MURST and GNSAGA of CNR (Italy).

\section{Definitions and tools}

We work over an algebraically closed field $\mathbf{K}$. We are interested in smooth projective curves, but we state the main definitions for any integral projective curve. For every integral projective curve $X$ let $M(X ; r, d)$ be the moduli scheme of stable torsion free sheaves, $E$, on $X$ with $\operatorname{rank}(E)=r$ and $\operatorname{deg}(E)=$ $d$. If $X$ is smooth of genus $g \geq 2$; then for all $r, d$ the scheme $M(X ; r, d)$ is smooth, connected and of dimension $r^{2}(g-1)+1$ ([9]). At least if $\operatorname{char}(\mathbf{K})=0$ for every smooth curve $X$ of genus $g$ and for all integers $r, d, t$ with $1 \leq t \leq$ $r-1$ the Lange invariant $s_{t}(E)$ of a general $E \in M(X ; r, d)$ is known and depends only on $r, t$ and $g$ : we have $s_{t}(E)=t(r-t)(g-1)+\varepsilon$, where $\varepsilon$ is the unique integer with $0 \leq \varepsilon \leq r-1$ and $\varepsilon+t(r-t)(g-1) \equiv t d \bmod (r)(\operatorname{see}[5]$, 4.6, or [7], Remark 3.14); we will call $s_{t}(r, d, g)$ this integer. In particular $s_{1}(r, d, g):=s_{1}(E)$ is the unique integer $s$ with $(r-1)(g-1) \leq s \leq(r-1) g$ and $s \equiv d \bmod (r)$. For the case $\operatorname{char}(\mathbf{K})>0$, see Remark 2.11. In [2], Def. 2.1, the following definition was introduced.

DEFINITION 1.1. Let $X$ be an integral projective curve and $E$ a rank $r$ torsion free sheaf on $X$. Assume $r \geq 2$. Fix a rank 1 subsheaf $L$ of $E$ computing $s_{1}(E)$, i.e. such that $\operatorname{deg}(L)$ is the maximal degree of a rank 1 saturated subsheaf of $E$. If $r=2$ we stop. Assume $r \geq 3$. Since $\operatorname{deg}(L)$ is maximal, $E / L$ is torsion free and $\operatorname{rank}(E / L)=r-1 \geq 2$. Let $L_{2}$ be a rank 1 subsheaf of $E / L$ computing $s_{1}(E / L)$. Equivalently, let $M_{2}$ be a rank 2 subsheaf of $E$ containing $L$ and with $\operatorname{deg}\left(M_{2}\right)=\operatorname{deg}(L)+\operatorname{deg}\left(L_{2}\right)$ maximal for all rank 2 subsheaves of $E$ containing $L$. Unfortunately, a priori the integer $\operatorname{deg}\left(L_{2}\right)$ may depend on the choice of $L$. However there is a unique integer, $t$, such that $t$ is the maximum of the integers $\operatorname{deg}\left(L_{2}\right)$ when $L$ vary among the saturated rank 1 subsheaves of $E$ with maximal degree. If $r=3$ we stop. Assume $r \geq 4$. We fix one such $L$ and one such $L_{2}=M_{2} / L$ with $(\operatorname{deg}(L), \operatorname{deg}(M 2 / L))$ maximal in the lexicographic order. Then consider a maximal degree rank 1 subsheaf, $T$, of $E / M_{2}$ with maximal degree; we choose $\left(L_{1}, M_{2}\right)$ such that $\operatorname{deg}(T)$ is maximal. And so on. At the end we obtain $r-1$ integers $\left(\operatorname{deg}\left(M_{1}\right), \ldots, \operatorname{deg}\left(M_{r-1}\right)\right)$ and 
an increasing filtration $\left\{M_{i}\right\}_{0 \leq i \leq r}$ of $E$ with $M_{0}=\{0\}, M_{r}=E$, each $M_{i}$, $1 \leq i \leq_{r-1}$, saturated in $M_{i+1}, \operatorname{rank}\left(M_{i}\right)=i$, and such that the ordered set of $r-1$ integers $\left(\operatorname{deg}\left(M_{1}\right), \ldots, \operatorname{deg}\left(M_{r-1}\right)\right)$ is maximal in the lexicographic order among all ordered set of $r-1$ integers obtained from such filtrations. We set $d_{i}(E):=\operatorname{deg}\left(M_{i+1}\right)-\operatorname{deg}\left(M_{i}\right), 1 \leq i \leq r-1$. By semicontinuity there is a Zariski open dense subset of $M(X ; r, d)$ on which the value $d_{i}(E)$ is constant and the $(r-1)$-ple of integers $\left(d_{1}(E), \ldots, d_{r-1}(E)\right)$ is minimal with respect to the lexicographic order. Set $d_{i}(r, d, g):=d_{i}(E)$ for general $E \in M(X ; r, d)$. For the value of $d_{i}(r, d, g)$ see the statement of Theorem 2.11. Notice that the integer $d_{i}(r, d, g)$ depends only on $r, d, g$ and $i$ but not on $X$. The integer $s_{i}(d, r, g)$ depends only on $r, d, g$ and $i$ but not on $X$. Just by their definitions $d_{1}(E)$ and $s_{1}(E)$ are equivalent invariants computed by the same subbundles of $E$, but this is usually not the case for $d_{i}(E)$ and $s_{i}(E)$ for $i \geq 2$, even for general $E$ (compare the values of $\sum_{1 \leq i \leq t} d_{i}(r, d, g)$ and $\left.s_{t}(r, d, g)\right)$.

The idea of the following key lemma is contained in [10], Prop. 1.11.

Lemma 1.2. Let $X$ be a reduced projective curve and $Q \in X_{\mathrm{reg}}$. Let

$$
0 \rightarrow H \rightarrow F \rightarrow G \rightarrow 0
$$

be an exact sequence of torsion free sheaves on $X$. Let $G^{\prime}\left(\right.$ resp. $\left.H^{\prime}\right)$ be obtained from $G$ making a positive (resp. negative) elementary transformation supported by $Q$. Then there exists a flat family $\left\{F_{u}\right\}_{u \in T}$ of torsion free sheaves on $X$ with $T$ integral quasi-projective curve and $o \in T$ such that $F_{o} \cong F$ and for every $u \in(T \backslash\{0\}) F_{u}$ fits in an exact sequence

$$
0 \rightarrow H^{\prime} \rightarrow F_{u} \rightarrow G^{\prime} \rightarrow 0
$$

and $F_{u}$ is obtained from $F$ making a negative elementary transformation supported by $Q$ and a positive elementary transformation supported by $Q$.

PRoof. Let $r$ (resp. $k$ ) be the rank of the restriction of $F$ (resp. $H$ ) to the unique irreducible component of $X$ containing $Q$, i.e. set $r:=\operatorname{dim}_{\mathbf{K}}(F \mid\{Q\})$ (resp. $\left.k:=\operatorname{dim}_{\mathbf{K}}(H \mid\{Q\})\right)$. There is a unique negative elementary transformation $f: F \rightarrow \mathbf{K}_{Q}$ of $F$ inducing the negative elementary transformation of $H$ which gives $H^{\prime}$. Set $F^{\prime}:=\operatorname{Ker}(f)$. Hence $F^{\prime}$ fits in an exact sequence

$$
0 \rightarrow H^{\prime} \rightarrow F^{\prime} \rightarrow G \rightarrow 0
$$

We may obtain $F^{\prime}$ from $F$ making a positive elementary transformation supported by $Q$ : just make the inverse of $f$. Let $\mathbf{v} \in\left(H^{\prime} \mid\{Q\}\right), \mathbf{v} \neq 0$, be a generator of the kernel of the linear map $H^{\prime}|\{Q\} \rightarrow H|\{Q\}$. Notice that $\mathbf{v}$ is 
a generator of the kernel of the linear map $F^{\prime}|\{Q\} \rightarrow F|\{Q\}$. Since $G^{\prime}$ is obtained from $G$ making a positive elementary transformation supported by $Q$, the linear map $G\left|\{Q\} \rightarrow G^{\prime}\right|\{Q\}$ has kernel of dimension 1. Call $\mathbf{z} \in G \mid\{Q\}$ a generator of this kernel. By the exact sequence (3) there is $\mathbf{w} \in\left(F^{\prime} \mid\{Q\}\right)$ whose image in $G \mid\{Q\}$ is $\mathbf{z}$. Since $\mathbf{z} \neq \mathbf{0}$, we have $\mathbf{w} \notin\left(H^{\prime} \mid\{Q\}\right)$. We will take as parameter space $T$ the affine line $K$ and as $o \in T$ the origin. For every $t \in \mathbf{K}$ set $\mathbf{v}_{t}:=\mathbf{v}+t \mathbf{w} \in F^{\prime} \mid\{Q\}$. Since $\mathbf{v} \in H^{\prime} \mid\{Q\}$ and $\mathbf{w} \notin H^{\prime}\left|\{Q\}, \mathbf{v}_{t} \in H^{\prime}\right|\{Q\}$ if and only if $t=0$ and $\mathbf{v}_{t} \neq 0$ for every $t \in \mathbf{K}$. Let $F_{t}^{\prime}$ be the sheaf obtained from $F^{\prime}$ making the positive elementary transformation supported by $Q$ and such that the kernel of the linear map $F^{\prime}\left|\{Q\} \rightarrow F^{\prime} t\right|\{Q\}$ is generated by $\mathbf{v}_{t}$. By construction $F_{0}^{\prime}=F$. If $t \neq 0, H^{\prime}$ is saturated in $F_{t}^{\prime}$ because $\mathbf{v}_{t} \notin H^{\prime} \mid\{Q\}$. Since for every $t \neq 0$ the image of $\mathbf{v}_{t}$ in $F^{\prime}\left|\{Q\} / H^{\prime}\right|\{Q\}$ is $\mathbf{w}$, for every $t \neq 0$ we have $F_{t}^{\prime} / H^{\prime} \cong G^{\prime}$, i.e. if $t \neq 0 F_{t}^{\prime}$ fits in an exact sequence (2), as wanted.

LEMMA 1.3. Let $X$ be an integral projective curve of arithmetic genus $g \geq 2$. Let (1) be a non-splitted exact sequence of torsion free sheaves on $X$ with $\operatorname{deg}(H)=0, \operatorname{deg}(G)=1, H$ semistable and $G$ stable. Then $E$ is stable.

Proof. In order to obtain a contradiction we assume the existence of a proper saturated subsheaf $F$ of $E$ with $\mu(F) \geq \mu(E)$. Since $\mu(E)=1 / \operatorname{rank}(E)>0$, we have $\operatorname{deg}(F)>0$. Hence $F$ is not contained in the semistable sheaf $H$, i.e. , calling $\pi: E \rightarrow H$ the quotient map, we have $F /(F \cap H) \cong \pi(F) \neq 0$. Since $F$ is stable of degree 1 and slope $1 / \operatorname{rank}(G)$, either $\pi(F)=G$ (and hence $\operatorname{deg}(\pi(F))=1)$ or $\operatorname{deg}(\pi(F)) \leq 0$. Since $H$ is stable and $\operatorname{deg}(H)=0$, either $H \subseteq F$ or $\operatorname{deg}(H \cap F)<0$ or $H \cap F=\{0\}$. If $H \cap F=\{0\}$ and $\pi(F)=G$, then (1) splits. Since $F \neq E$ and $\operatorname{deg}(F)>0$, we obtain a contradiction.

Definition 1.4. Let $X$ be an integral projective curve, $Q \in X_{\text {reg }}$ and $E$ a rank $r$ torsion free sheaf on $X$. Set $t(E, Q):=\inf \{t \in Z, t \geq 0$ : there exists a stable rank $r$ sheaf $F$ obtained from $E$ making $t$ positive elementary transformations supported by $Q\}, t(E, Q,+):=\inf \{t \in Z, t \geq 0$ : for every integer $u \geq t$ there exists a stable rank $r$ sheaf $F$ obtained from $E$ making $u$ positive elementary transformations supported by $Q\}, t(E):=\inf \{t \in$ $Z, t \geq 0$ : there exists a stable rank $r$ sheaf $F$ obtained from $E$ making $t$ positive elementary transformations supported by smooth points of $X$ \} and $t(E,+):=\inf \{t \in Z, t \geq 0$ : for every integer $u \geq t$ there exists a stable rank $r$ sheaf $F$ obtained from $E$ making $t$ positive elementary transformations supported by smooth points of $X$ \}.

DEFINITION 1.5. Make the same definition as in 1.4 just taking negative elementary transformations instead of positive elementary transformations and call $t(E, Q)^{\prime}, t(E, Q,+)^{\prime}, t(E)^{\prime}$ and $t(E,+)^{\prime}$ the corresponding integers. 
Remark 1.6. Assume that $X$ is Gorenstein. A torsion free sheaf $E$ is stable if and only if $E^{*}$ is stable (see e.g. [2]). Hence if $X$ is Gorenstein we have $t(E, Q)^{\prime}=t\left(E^{*}, Q\right), t(E, Q,+)^{\prime}=t\left(E^{*}, Q,+\right), t(E)^{\prime}=t\left(E^{*}\right)$ and $t(E,+)^{\prime}=t\left(E^{*},+\right)$.

REMARK 1.7. By the openness of stability in the definitions of the integers $t(E, Q), t(E, Q,+), t(E)$ and $t(E,+)$ made in 1.4 we may assume that the corresponding positive elementary transformations are general.

REMARK 1.8. If in the definition of the integers $t(E, Q), t(E, Q,+), t(E)$ and $t(E,+)$ made in 1.4 we use the word "semistable" instead of the word "stable" we obtain certain integers, say $t(E, Q)$ ", $t(E, Q,+)$ ", $t(E)$ " and $t(E,+)^{\prime \prime}$. Using these integers it is obvious how to modify the statements of 1.2 and 1.9 to obtain semistable sheaves instead of stable sheaves. For semistability we have also a modification of 1.10 below in which we assume $\operatorname{deg}(H)=-\operatorname{deg}(G)$ (using instead of 1.3 the obvious fact that for two semistable sheaves, say $H^{\prime \prime}$ and $G^{\prime \prime}$, with $\operatorname{deg}\left(H^{\prime \prime}\right)=\operatorname{deg}\left(G^{\prime \prime}\right)=0$, the sheaf $H^{\prime \prime} \oplus G^{\prime \prime}$ is semistable).

Remark 1.9. Note that for every $L \in \operatorname{Pic}(X)$ and any $E$ we have $t(E \otimes$ $L, Q)=t(E, Q), t(E \otimes L, Q,+)=t(E, Q+), t(E \otimes L)=t(E)$ and $t(E \otimes L,+)=t(E,+)$.

Theorem 1.10. Fix integers $r, k, t$ with $r>k>0$ and $t \geq 0$. Let $X$ be an integral projective curve. Let $H$ and $G$ be torsion free sheaves on $X$ with $\operatorname{rank}(H)=k, \operatorname{rank}(G)=r-k, \operatorname{deg}(H)=-t, \operatorname{deg}(G)=t+1, t(H,+) \leq t$, $t(G,+)^{\prime} \leq t$. Then there is an exact sequence (1) with E stable.

Proof. By the assumptions on $t(H,+)$ and $t(G,+)^{\prime}$ and Remark 1.7 there exists a stable sheaf $H^{\prime \prime}$ (resp. $G^{\prime \prime}$ ) of degree 0 (resp. 1) obtained from $H$ (resp. $G)$ making $t$ positive (resp. negative) elementary transformations supported

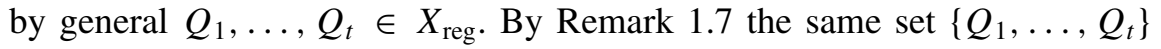
may be used both by the positive and negative elementary transformations. By Lemma 1.3 there is an exact sequence

$$
0 \rightarrow H^{\prime \prime} \rightarrow E^{\prime \prime} \rightarrow G^{\prime \prime} \rightarrow 0
$$

with $E^{\prime \prime}$ stable. We apply Lemma $1.2 t$ times to (4) and use the choice of $H^{\prime \prime}$ and $G^{\prime \prime}$ to conclude. 


\section{The main results}

In the first part of this section we start with a direct sum of $r-1$ line bundles to obtain rank $r$ stable vector bundles, $E$, with suitable $s_{t}(E)$ and $d_{t}(E)$ and for which we may control the set of rank $t$ subbundles of $E$ computing $s_{t}(E)$ and $d_{t}(E)$ (see Theorems 2.2 and 2.5). Then we consider the same problem starting from an extension of a general vector bundle $G$ by a general vector bundle $H$ (see Theorems 2.6, 2.7, 2.8 and 2.9).

TheOREM 2.1. Let $X$ be a smooth curve of genus $g \geq 2$. Fix an integer $r \geq 2$ and integers $a(i), 1 \leq i \leq r$; set $z:=\max _{1 \leq j \leq r-1}\{a(j)\}$ and assume $a(r)>-\sum_{1 \leq j \leq r-1} a(j)+(r-1) z$. Fix $L_{j} \in \operatorname{Pic}^{a(j)}(X), 1 \leq j \leq r-1$; assume that no two among the line bundles $L_{j}$ 's with $a(j)=z$ are isomorphic; set $F:=\bigoplus_{1 \leq j \leq r-1} L_{j}$. Then for a general $L_{r} \in P i c^{a(r)}(X)$ the general extension of $L_{r}$ by $F$ is stable.

Proof. Take $R \in \operatorname{Pic}^{z}(X)$ with $R$ not isomorphic to any line bundle $L_{j}$, $1 \leq j \leq r-1$, with $a(j)=z$. Set $U:=F \oplus R$. It is sufficient to prove that the general bundle obtained from $U$ making $t$ elementary transformations is stable. By the openness of stability it is sufficient to prove this assertion when the first $-\sum_{1 \leq j \leq r-1} a(j)+(r-1) z$ positive elementary transformations of $U$ are very particular. For each integer $j$ with $1 \leq j \leq r-1$ and $a(j)<z$ we will do $z-a(j)$ positive elementary transformations of $\mathrm{U}$ which increase the degree of the factor $L_{j}$ of $U$. Since $g>0$ we may do this and arrive at a bundle, $R \oplus F^{\prime}, F^{\prime} \cong \bigoplus_{1 \leq j \leq r-1} L_{j}^{\prime \prime}$ with $L_{j}^{\prime \prime} \in P i c^{z}(X)$ and such that no two among the line bundles $L_{j}^{\prime \prime}$ are isomorphic or isomorphic to $R$, i.e. we may reduce to the case in which $a(j)=0$ for every $j$ with $1 \leq j \leq r-1$. This case is a particular case of Lemma 1.3 (or see [1], Prop. 2.3).

TheOREM 2.2. Let $X$ be a smooth curve of genus $g \geq 2$. Fix an integer $r \geq 2$ and integers $a(i), 1 \leq i \leq r$, with $a(i) \leq a(j)$ for $1 \leq i \leq j \leq s, a(r-1) \leq 0$, $a(r)>-\sum_{1 \leq j \leq r-1} a(j)+(r-1) a(r-1)$ and $a(r)-a(1) \leq g-2$. Fix general $L_{i} \in \operatorname{Pic}^{a(i)}(X), 1 \leq i \leq r$ and set $F:=\bigoplus_{1 \leq j \leq r-1} L_{j}$. Let $E$ be the general extension of $L_{r}$ by $F$. Then $E$ is stable and for every integer $k$ with $1 \leq j \leq k$ we have $s_{k}(E)=-\sum_{r-k \leq j \leq r-1} a(j)$. Furthermore, for every integer $k$ with $1 \leq k \leq r-1$ every rank $k$ subbundle of $E$ computing $s_{k}(E)$ is the direct product of $k$ of the line bundles $L_{j}, 1 \leq j \leq r-1$, with degrees $a(r-k), \ldots, a(r-1)$; in particular if either $k=r-1$ or $a(r-k-1)<a(r-k)$, then $\bigoplus_{r-k \leq j \leq r-1} L_{j}$ is the unique rank $k$ subbundle of $E$ which computes $s_{k}(E)$.

Proof. The stability of $E$ was proved in 2.1. We divide the proof of the other assertions into 4 steps. Set $F^{\prime}:=\bigoplus_{2 \leq j \leq r-1} L_{j}$. 
Step 1. We claim that for every integer $x$ with $1 \leq x \leq r-2$ we have $s_{x}(F)=-\sum_{r-x \leq j \leq r-1} a(j)$ and that every rank $x$ subbundle of $F$ which compute $s_{x}(F)$ is the direct sum of $x$ line bundles $L_{j}, 1 \leq j \leq r-1$, of degrees $a(r-x), \ldots, a(r-1)$; in particular we claim that if $a(r-x-$ 1) $<a(r-x)$, then $\bigoplus_{r-x \leq j \leq r-1} L_{j}$ is the unique rank $x$ subbundle of $F$ which computes $s_{x}(F)$. Let $T$ be a subsheaf of $F$ which computes $s_{x}(F)$. Since every $L_{j}$ is general and $\left|\operatorname{deg}\left(L_{i}\right)-\operatorname{deg}\left(L_{j}\right)\right| \leq g-2$ for all $i, j$, we have $h^{0}\left(X, \operatorname{Hom}\left(L_{i}, L_{j}\right)\right)=0$ for all pairs $i, j$ with $i \neq j$. Hence $H^{0}(X, \operatorname{End}(F)) \cong \bigoplus_{1 \leq j \leq r-1} H^{0}\left(X, \operatorname{End}\left(L_{j}\right)\right) \cong \mathbf{K}^{\oplus(r-1)}$ and $\operatorname{Aut}(F) \cong$ $\left(\mathbf{K}^{*}\right)^{\oplus(r-1)}$ is represented by the diagonal matrices. For every vector bundle $A$ on $X$ and for every integer $x$ with $1 \leq x<\operatorname{rank}(A)$ the set $M_{x}(A)$ of all rank $x$ subsheaves of $A$ with maximal degree is a complete subscheme of the Quot-scheme Quot $(A)$ of $A$. Hence, taking a one-parameter subgroup $\left\{t_{\lambda}\right\}_{\lambda \in \mathbf{K}^{*}}$ of $\operatorname{Aut}(F)$, and taking a limit inside $\operatorname{Quot}(F)$ of the flat family $\left\{t_{\lambda}(T)\right\}_{\lambda \in \mathbf{K}^{*}}$ for $\lambda$ going to 0 , we reduce to the case in which either $T \cap L_{1}=\{0\}$ or $L_{1}$ is a direct factor of $T$. First assume $T \cap L_{1}=\{0\}$. Hence $T$ has a isomorphic image, $T^{\prime}$, under the projection $F \rightarrow F^{\prime}$. If $x=r-2$, we have $T \cong F^{\prime}$. Since $h^{0}\left(X, \operatorname{Hom}\left(F^{\prime}, L_{1}\right)\right)=h^{0}\left(X, \operatorname{Hom}\left(L_{1}, F^{\prime}\right)\right)=0$ we obtain $T=F^{\prime}$, as wanted. If $x \leq r-3$, we apply induction on $r$ to the pair $\left(F^{\prime}, T^{\prime}\right)$ and conclude. Now assume that $L_{1}$ is a direct factor of $T$, say $T=L_{1} \oplus T^{\prime}$. We apply the inductive assumption on $r$ to the pair $\left(F^{\prime}, T^{\prime}\right)$.

Step 2. Fix an integer $k$ with $1 \leq k \leq r-1$ and a rank $k$ subbundle, $H$, of $E$ computing $s_{k}(E)$. By the claim proved in Step 1 it is sufficient to check that $H$ is a subsheaf of $F$. From now on, we assume that this is not true and call $L \cong$ $H /(H \cap F)$ the image of $H$ in $L_{r}=E / F$. By assumption $L \in \operatorname{Pic}(X)$ and there is an effective Cartier divisor $D$ such that $L \cong L_{r}(-D)$. Set $d:=\operatorname{deg}(D) \geq 0$. Since $a(r)-a(1) \leq g-2$ and $\operatorname{deg}(H)=\operatorname{deg}(H \cap F)+\operatorname{deg}(L)$, we may assume $d \leq g-2$. Since $g \geq 2$ we have $\operatorname{dim}\left(W_{d}^{1}(X)\right) \leq d-1$ and hence, for fixed $L_{r}$, the set of all possible $D$ depends on at most $d$ parameters. By Riemann-Roch and Serre duality we have $\operatorname{dim}\left(\operatorname{Ext}^{1}\left(X, L_{r}, F\right)\right)=\sum_{1 \leq j \leq r-1}(a(r)-a(j))+$ $(r-1)(g-1)$.

Step 3. Here we assume $x=1$, i.e. $H \cong L$. Set $T:=\{\operatorname{rank} r$ vector bundles, $M$, on $X$ such that $\operatorname{deg}(M)=\operatorname{deg}(E), M$ containg $F \oplus L, F$ is saturated in $M$ and $M / F \cong L r\}$. If $d=0$, then $L=L_{r}$ and $T=\{F \oplus L\}$. If $d>0$ the set $T$ is parametrized by an irreducible variety of dimension $r d-1$. For fixed $L_{r}$, the set of possible divisors $D$ and hence the set of possible isomorphism classes of line bundles $L \cong L_{r}(-D)$ depends on at most $d$ parameters. Note that for every $E$ there is at most an embedding of $F$ in $E$ (up to an element of $\operatorname{Aut}(F)$ ) (use for instance that $\left.h^{0}\left(X, \operatorname{Hom}\left(F, L_{r}\right)\right)=0\right)$. Since $d \leq a(r)-a(r-1)$, we obtain a contradiction.

Step 4. From now on, we assume $x \geq 2$. The vector space $\operatorname{Ext}^{1}\left(X, L_{r}, F^{\prime}\right)$ 
is a direct factor of the vector space $E x t^{1}\left(X, L_{r}, F\right)$. Hence every extension of $L_{r}$ by $F^{\prime}$ lifts to an extension of $L_{r}$ by $F$. Call $\pi: \operatorname{Ext}^{1}\left(X, L_{r}, F\right) \rightarrow$ $\operatorname{Ext}^{1}\left(X, L_{r}, F^{\prime}\right)$ the corresponding surjection. Fix e $\in \operatorname{Ext}^{1}\left(X, L_{r}, F\right)$ and call $E$ its middle term. The middle term, $E^{\prime}$, of $\pi(\mathbf{e})$ is obtained from the extension e by the map $F \rightarrow F^{\prime}$. Hence e and $\pi(\mathbf{e})$ fit in a commutative diagram of short exact sequences and by the snake lemma the induced map $f: E \rightarrow E^{\prime}$ is surjective. Set $H^{\prime}:=f(H) \subset E^{\prime}$. If $H \cap L_{1}=\{0\}$, we have $H^{\prime} \cong H$ and we conclude by induction on $r$ because $\pi$ is surjective and hence $\pi(\mathbf{e})$ is general if e is general. If $H \cap L_{1} \neq \emptyset$, by the inductive assumption on $r$ applied to the data $\left(F^{\prime}, L_{r}\right)$ we have $\operatorname{deg}\left(H^{\prime}\right) \geq-\sum_{x<j \leq r-1} a(j)$. Since $\operatorname{deg}\left(H \cap L_{1}\right) \leq a(1)$ with strict inequality unless $H \cap L_{1}=L_{1}$, we obtain $\operatorname{deg}(H)<\operatorname{deg}\left(\bigoplus_{r-k \leq j \leq r-1} L_{j}\right)$ (and hence a contradiction), unless $a(1)=$ $a(r-x)$ and $H$ contains $L_{1}$. We need to check that if this is the case, then $H \cong L_{1} \oplus H^{\prime}$ or that one of the previous assumptions leads to a contradiction. By the inductive assumption $H^{\prime}$ is a direct sum of $x-1$ line bundles $L_{j}$ with $j>1$ and it is contained in $F^{\prime}$. Hence $H$ is contained in $F$, contradiction.

REMARK 2.3. If some of the line bundles $L_{j}, 1 \leq j \leq r-1$, have the same degree but are not isomorphic we obtain a stable bundle $E$ which has, for a certain integer $t$, a perfectly described set, $\mathbf{S}$, of maximal degree rank $t$ subbundles with $\operatorname{card}(\mathbf{S})>1$ and $\operatorname{dim}(\mathbf{S})=0$. Now assume that a of the line bundles $L_{j}$ are isomorphic; we obtain a vector bundle $E$ such that the corresponding set $\mathbf{S}$ is perfectly described and $\operatorname{dim}(\mathbf{S}) \geq 1$. We need to show that such bundle $E$ is stable. For the stability of $E$ we add the condition $a(r)>$ $-\sum_{1 \leq j \leq r-1} a(j)+(r-1) a(r-1)+a$ to be able to quote [1], Cor. 2.4, instead of [1], Prop. 2.3, at the end of the proof of Theorem 2.1. The same observation works for the integers $d_{t}(E)$ in the set-up of Theorem 2.5 below.

TheOREM 2.4. Let $X$ be a smooth curve of genus $g \geq 2$. Fix an integer $r \geq 2$ and integers $a(i), 1 \leq i \leq r$, with $a(i) \leq a(j)$ for $1 \leq i \leq j \leq s$, $a(r-1) \leq 0, a(r)>-(r-1) a(r-1)$ and $a(r)-a(1) \leq g-2$. Fix general $L_{i} \in \operatorname{Pic}^{a(i)}(X), 1 \leq i \leq r$ and set $F:=\bigoplus_{1 \leq j \leq r-1} L_{j}$. Let $E$ be the general extension of $L_{r}$ by $F$. Then for every integer $k$ with $1 \leq j \leq k$ we have $d_{k}(E)=-\sum_{r-k \leq j \leq r-1} a(j)$. For every integer $k$ with $1 \leq k \leq r-2$ and such that $a(r-k-1)<a(r-k)$ the bundle $\bigoplus_{r-k<j \leq r-1} L_{j}$ is the unique rank $k$ subbundle of $E$ which computes $d_{k}(E) . F$ is the unique rank $r-1$ subbundle of $E$ which computes $d_{r-1}(E)$. If we assume $a(r)>-\sum_{1 \leq j \leq r-1} a(j)+(r-$ 1) $a(r-1)$, then $E$ is stable.

Proof. The last assertion is a particular case of Theorem 2.1. To prove the first assertion we use induction on $r$, the case $r=2$ being [8], Prop. 3.3. Assume $r \geq 3$ and let $L$ be a rank 1 subbundle of $E$ computing $d_{1}(E)$. 
Notice that $\operatorname{deg}(L) \geq a(r-1)$. We have to check that $L$ is one of the direct factors of $F$, i.e. that $L$ is contained in $F$. Assume that this is not true. Then we obtain a non-zero map $L \rightarrow L_{r}$, i.e. an effective divisor $D$ with $d:=\operatorname{deg}(D)=a(r)-d_{1}(E) \leq a(r)-a(1)$. Since $g \geq 2$ we have $\operatorname{dim}\left(W_{d}^{1}(X)\right) \leq d-1$ and hence, for fixed $L_{r}$, the set of all possible $D$ depends on at most $d$ parameters. By Riemann-Roch and Serre duality we have $\operatorname{dim}\left(\operatorname{Ext}^{1}\left(X, L_{r}, F\right)\right)=\sum_{1 \leq j \leq r-1}(a(r)-a(j))+(r-1)(g-1)$. Now we copy verbatim Step 3 of the proof of 2.2.

We will use the "generic values" $s_{t}(x, y, g)$ of the Lange invariants to compute the Lange invariants of certain stable vector bundles. Here we would like to compute the Lange invariants of the middle term of an exact sequence (1).

THEOREM 2.5. Assume char $(\mathbf{K})=0$. Fix integers $g, r, k, t, a, b$ with $g \geq 2$, $1 \leq t<k<r / 2, a / k<b /(r-k)$ and $s_{t}(k, a, g)<s_{t}(r-k, b, g)$. Let (1) be the general extension of a general $G \in M(X ; r-k, b)$ by a general $H \in M(X ; k, a)$. Then $F$ is stable and $s_{t}(F)=s_{t}(k, a, g)$. Furthermore, every rank $t$ subbundle of $F$ computing $s_{t}(F)$ is contained in $H$.

Proof. The stability of $F$ was proved in [10]. Since $H$ is general, we have $s_{t}(H)=s_{t}(k, a, g)$ and hence it is sufficient to prove the last assertion. Assume the existence of a rank $k$ subbundle $M$ of $F$ computing $s_{t}(F)$ but not contained in $H$. Let $N \neq 0$ be the saturation of the image of $M$ in $G=F / H$. First assume $M \cap H=\{0\}$. Hence $\operatorname{rank}(N)=t$ and $\operatorname{deg}(N) \geq \operatorname{deg}(M)$. Since $t b-(r-k) \operatorname{deg}(N) \leq s_{t}(r-k, b, g)<s_{t}(k, a, g)=s_{t}(H)$, we obtain a contradiction. Now assume $N \cap H \neq\{0\}$ and set $\rho:=\operatorname{rank}(M \cap H)$. Hence $1 \leq \rho<t$ and $\operatorname{rank}(N)=t-\rho$. Since $\operatorname{deg}(M) \leq \operatorname{deg}(N)+\operatorname{deg}(M \cap H)$, $\rho a-k(\operatorname{deg}(M \cap H)) \geq s_{r}(k, \rho, g)$ and $(t-\rho) b-(r-k)(\operatorname{deg}(N)) \geq$ $s_{t-r}(r-k, b, g)$, we obtain a contradiction.

Theorem 2.6. Assume $\operatorname{char}(\mathbf{K})=0$. Fix integers $g, r, a, b$ with $g \geq 2$, $r \geq 3, a<b /(r-1)$ and $(r-1) a<b+(r-2)(g-1)$. Let $(1)$ be the general extension of a general $G \in M(X ; r-1, b)$ by a general $H \in \operatorname{Pic}^{a}(X)$. Then $F$ is stable and $s_{1}(F)=a$. Furthermore, $H$ is the unique rank 1 subbundle of $F$ computing $s_{1}(F)$.

Proof. The stability of $F$ is a very particular case of the main result of [10]. Assume the existence of a rank 1 subsheaf $L$ of $F$ with $\operatorname{deg}(L) \geq a$ and $L \neq H$. Hence the saturation, $L^{\prime}$, of the image of $L$ in $G:=F / H$ is a rank 1 subbundle of degree $\geq \operatorname{deg}(L) \geq a$. Since $G$ is general, we have $\mu\left(L^{\prime}\right) \leq \mu\left(G / L^{\prime}\right)-(g-1)$ and hence $a \leq b /(r-2)-(g-1)$, contradiction.

The same proof gives the following two results. 
TheOREM 2.7. Assume char $(\mathbf{K})=0$. Fix integers $g, r, k, a, b$ with $g \geq 2$, $1 \leq t<k<r / 2, a / k<b /(r-k)$ and $a<s k(r-k, b, g)$. Let (1) be the general extension of a general $G \in M(X ; r-k, b)$ by a general $H \in M(X ; k, a)$. Then $F$ is stable and $s_{k}(F)=a$. Furthermore, $H$ is the unique rank $k$ subbundle of $F$ computing $s_{k}(F)$ is contained in $H$.

Theorem 2.8. Assume char $(\mathbf{K})=0$. Fix integers $g, r, k, t, a, b$ with $g \geq 2$, $1 \leq k \leq r-1,1 \leq t<k<r / 2, a / k<b /(r-k)$ and $d_{t}(k, a, g)>d_{t}(r-$ $k, b, g)$. Let (1) be the general extension of a general $G \in M(X ; r-k, b)$ by $a$ general $H \in M(X ; k, a)$. Then $F$ is stable and $d_{t}(F)=d_{t}(k, a, g)=d_{t}(H)$. Furthermore, every rank $t$ subbundle of $F$ computing $d_{t}(F)$ is contained in $H$.

Theorem 2.9. Fix integers $g, r, d$ with $g \geq 2$ and $r \geq 2$. Define the integers $d_{k}(r, g), 1 \leq k \leq r-1$, in the following way. Let $d_{1}(r, d, g)$ be the unique integer $s$ with $(r-1)(g-1) \leq s \leq(r-1) g$ and $s \equiv d \bmod (r)$. Set $D_{1}(r, d, g):=d-d_{1}(r, d, g)$. Assume $2 \leq k \leq r-1$ and that we have defined inductively the integers $d_{j}(u, x, g)$ and $D_{j}(r, d, g)$ for all integers $j$ with $1 \leq j \leq k-1$, all integers $u$ with $j<u \leq r$ and all integers s. Set $d_{k}(r, d, g):=d_{1}\left(r-k+1, D_{k-1}(r, d, g), g\right)+d_{k-1}(r, d, g)$ and $D_{k}(r, d, g):=$ $D_{k-1}(r, d, g)-d_{1}\left(r-k+1, D_{k-1}(r, d, g), g\right)$. Let $X$ be a smooth projective curve of genus $g$. Then for a general $E \in M(X ; r, d)$ we have $d_{k}(E)=$ $d_{k}(r, d, g)$ for every integer $k$ with $1 \leq k \leq r-1$.

Proof. The value of $d_{1}(r, d, g)$ is just the value of $s_{1}(E)=d_{1}(E)$ by [7], Cor. 3.13 and the discussion between 3.13 and 3.14. If $r=2$, we have finished; anyway, in this case the result was very well-known ([8]). Assume $r \geq 3$. Take a rank 1 subbundle $L$ of $E$ with $\operatorname{deg}(E)=d_{1}(r, d, g)$ and set $A:=E / L$. We have $\operatorname{rank}(A)=r-1$ and $\operatorname{deg}(A)=d-d_{1}(r, d, g)=D_{1}(r, d, g)$. We claim that for general $E, A$ is general. This follows from the main result of [10] and the discussion made in [6] of the conjecture solved in [10]. Note that, for suitable $L$, we have $d_{2}(E)=d_{1}(A)$. To obtain $d_{2}(E)=d_{2}(r, d, g)$ it is sufficient to use the claim, the definitions of the integer $D_{1}(r, d, g)$ and apply the case $k=1$ proved at the beginning to the vector bundle $A$. If $r=3$, we have finished. If $r>3$ the same inductive proof and the inductive definitions of the integers $d_{k}(E)$ and $d_{k}(r, d, g)$ give the result for all values of $k$.

Remark 2.10. Assume $\operatorname{char}(\mathbf{K})>0$. To have 2.6, 2.7, 2.8 and 2.9 it is sufficient to obtain for all $r, d$ and $t$ the same values for the integers $s_{t}(E)$, $E$ general in $M(X ; r, d)$ as in the characteristic 0 case and to be able to use freely [10]. It seems that the proof given in [10], Th. 1.2, of the unpublished result [5], works in any characteristic, modulo the quotation of [6], 2.1 and 2.2. Using the main result of [10] for lower ranks, it seems possible to cover everything in positive characteristic, but we do not have checked it. 


\section{REFERENCES}

1. Ballico, E., Brill-Noether theory for vector bundles on projective curves, Math. Proc. Camb. Phil. Soc. 124 (1998), 483-499.

2. Ballico, E., Stable sheaves on reduced projective curves, Ann. Mat. Pura Appl. 175 (1998), 375-383.

3. Ballico, E., Extensions of stable vector bundles on smooth curves: Lange's conjecture, An. Stiint. Univ. Al. I, Cuza Iasi. Mat. (N.S.) 46 (2000), 149-156.

4. Brambila-Paz, L. and Lange, H., A stratification of the moduli space of vector bundles on curves, J. reine angew. Math. 494 (1998), 173-187.

5. Hirschowitz, A., Probléme de Brill-Noether en rang supérieur, Prepublication Mathématiques n. 91, Nice, 1986.

6. Lange, H., Zur Klassifikation von Regelmanigfaltigkeiten, Math. Ann. 262 (1983), 447-459.

7. Lange, H., Some geometrical aspects of vector bundles on curves, Aportaciones Matematicas Notas de Investigacion 5 (1992), 53-74.

8. Lange, H. and Narasimhan, M. S., Maximal subbundles of rank 2 vector bundles on curves, Math. Ann. 266 (1983), 55-72.

9. Newstead, P. E., Introduction to moduli problems and orbit spaces, Tata Inst. Lecture Notes, Springer-Verlag, 1978.

10. Russo, B. and Teixidor i Bigas, M., On a conjecture of Lange, J. Algebraic Geometry 26 (1998), 483-496.

11. Teixidor i Bigas, M., Unicity of the Lange subbundle, preprint.

DEPT. OF MATHEMATICS,

UNIVERSITY OF TRENTO

38050 POVO (TN)

ITALY

E-mail: ballico@science.unitn.it 